\title{
Surgical relief of esophageal compression by thoracic aorta
}

\author{
Ming-Ho $\mathrm{Wu}^{*}$ and Han-Yun Wu \\ Department of Surgery, Tainan Municipal Hospital, 670 Chung-Te Rd, Tainan, Taiwan
}

\begin{abstract}
We describe surgical procedures of separation of the distal esophagus from the aorta, transposition of the distal esophagus and cardia, creation of new hiatus, and splenectomy to relief esophageal compression by tortuous aorta. The patient can swallow regular diet after surgical intervention.
\end{abstract}

\section{Introduction}

A 74-year-old woman suffered from dysphagia for 6 months. She had hypertension and atrial fibrillation for more than 5 years. Chest computed tomography and esophagography showed the tortuous aorta compressing the esophagus (Figure 1). To solve the problem of dysphagia, a three dimension reconstruction from computed tomography was performed and surgical procedures were planned to release compression of the esophagus from the aorta, heart, and spleen (Figure 2). Operative images and postoperative esophagography (Figure 3) showed the esophageal compression completely relieved.

\section{Technique}

The patient was put on supine position with left side 30-degree elevation after general anesthesia using one-lung ventilation. The surgical procedures were as following; a $12-\mathrm{cm}$ incision was made via left 6th ICS to subcostal area, separation of the distal esophagus from the aorta, transposition of the distal esophagus and cardia, creation of new hiatus, and splenectomy were performed. Separation of distal esophagus from the aorta was carried out up to the level of left inferior pulmonary vein. The esophagus and cardia were mobilized free from the hiatus. A new hiatus was moved toward left anterior aspect. The esophagus was fixed to the new hiatus using sutures. Previous hiatus was closed using non-absorbable sutures. During the procedure, a 42 Fr. Maloney Dilator Bougie was placed in the esophagus to keep a suitable new hiatus.

\section{Comments}

Dysphagia aortica is difficulty in swallowing caused by extrinsic compression of the esophagus due to an ectatic, tortuous, or aneurysmatic atherosclerotic thoracic aorta. This condition is very uncommon, and it is usually associated with old age, women with short stature, hypertension, and kyphosis. It should be differentiated from other causes of dysphagia, such as gastroesophageal reflux disease or motility disorders, because dysphagia aortica often requires surgical intervention that can significantly reduce the morbidity and these interventions can be curative in some situations [1]. Some patients with dysphagia aortica were delayed in diagnosis, even got fatal outcome $[2,3]$. Surgical intervention consists of aortic stent for aortic aneurism, aortic resection and graft, or esophageal transposition. Lambert [4]

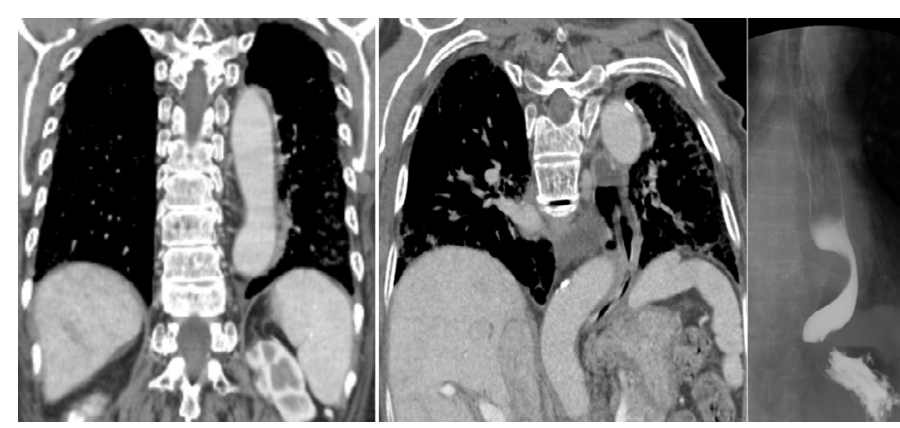

Figure 1. CT revealed the descending aorta lied on left side of the vertebra (left) and then decussated to in front of the vertebra (middle) that resulted in compression of the esophagus (right).
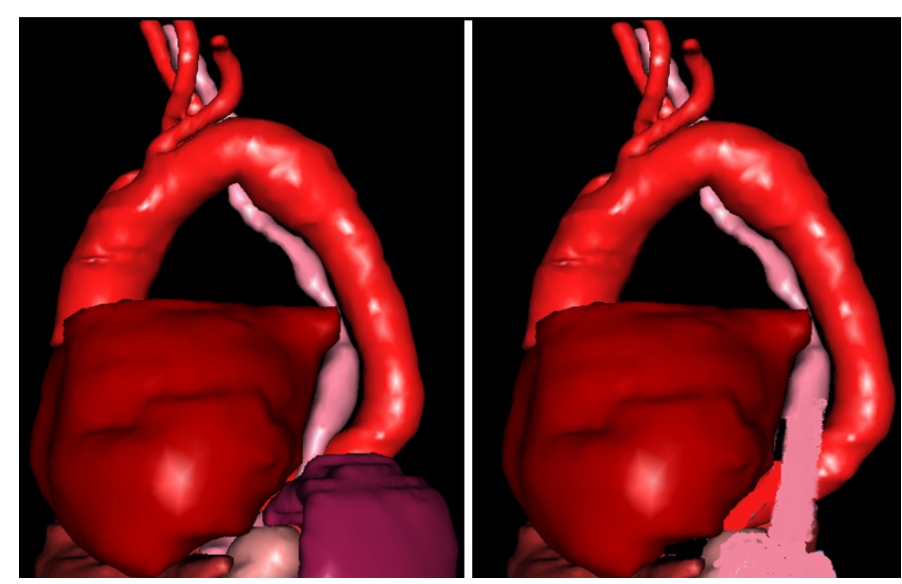

Figure 2. Three dimension reconstruction from computed tomography revealed the relationship of the esophagus to the heart, aorta, and the spleen (left), and a new way of the distal esophagus was planned (right).

Correspondence to: Ming-Ho Wu, Department of Surgery, Tainan Municipal Hospital, 670 Chung-Te Rd, Tainan, Taiwan, Tel: 886-6-2609926; Fax: 886-62606351; E-mail: m2201@mail.ncku.edu.tw

Key words: esophageal compression, dysphagia, tortuous aorta

Received: March 11, 2017; Accepted: April 17, 2017; Published: April 20, 2017 

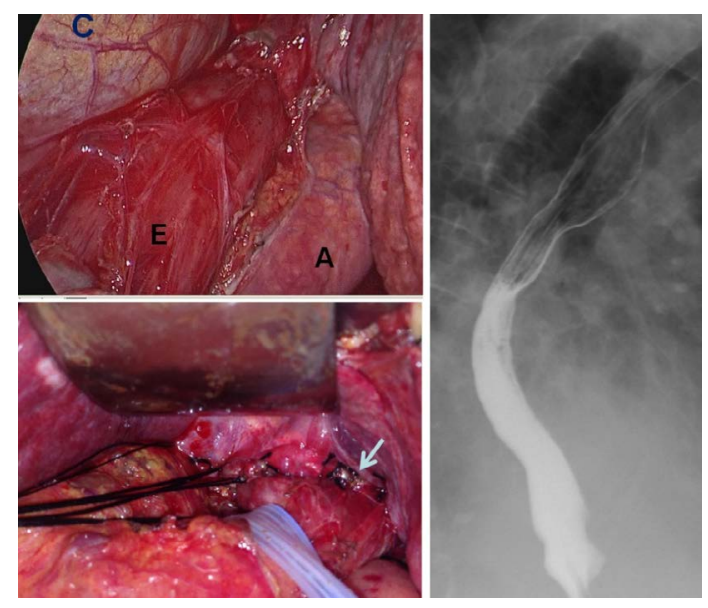

Figure 3. Operative images (left) showed the mobilized esophagus (E) related to the heart (C) and aorta (A), and new hiatus (arrow). Postoperative eshophagography showed normal patency of the esophagus (right). described the surgical correction of esophageal obstruction due to tortuosity of the aorta. In order to get a good result in our patient, we performed three dimension images from the contrast computed tomography that we choice the suitable surgical procedures. The patient can swallow regular diet following surgical intervention. In conclusion, patients with tortuous aorta require the study of esophagography if presence of swallowing difficulty. Surgical intervention could be considered.

\section{References}

1. Kim JH, Jang SW, Kim DB, Lee HJ, Kim JG, et al. (2009) A Patient With Dysphagia due to an Aortic Aneurysm. Korean Circ J 39: 258-260. [Crossref]

2. Rajpoot M (2016) Dysphagia aortica: Systematic review of a fatal disease JMPR 3: 10-11.

3. Liao CY, Huang SC, Wang YC, Chin HK, Tsai CC, et al. (2015) Dysphagia aortica: a fatal delay in diagnosis. Am J Emerg Med 33: 1117. [Crossref]

4. Lambert A (1971) Surgical correction of esophageal obstruction due to tortuosity of the aorta. Case report describing relief by means of a new operative procedure. $J$ Thorac Cardiovasc Surg 62: 973-978. [Crossref]

Copyright: (C2017 Wu MH. This is an open-access article distributed under the terms of the Creative Commons Attribution License, which permits unrestricted use, distribution, and reproduction in any medium, provided the original author and source are credited. 